\title{
106. A New Model of the Chromosphere
}

\author{
By Zenzaburo Suemoto \\ Tokyo Astronomical Observatory \\ (Comım. by Yusuke Hagihara, M.J.A., Sept. 12, 1963)
}

1. Introduction. About a decade ago the normal solar chromosphere was supposed to be a more or less uniform atmosphere, although there are some minor irregularities as can be seen on spectroheliograms. In 1956 Athay and Menzel $^{11}$ proposed a non-uniform model with a high temperature region emitting helium lines, covering a low temperature region emitting hydrogen and metallic lines. However, in 1958 Athay $^{22}$ found that with this model, the solar radio emission had to be more intense than is observed. In 1961 Moriyama $^{3)}$ investigated this problem again and suggested that with a helium and radio emitting layer covering the solar disk uniformly one cannot escape from this contradiction. He finally suggested that the helium and radio emitting region does not cover the whole disk but occupies only one tenth of it. In 1962 Suemoto and Moriyama ${ }^{4)}$ compared the extreme ultraviolet radiation with radio emission and arrived at the similar conclusion that the transition layers with temperatures intermediate between the chromosphere and the corona also occupy only one tenth of the solar disk. However, these arguments refer only to high temperature regions presumably surrounding spicules and do not refer to the starting level of spicules themselves.

Suemoto and $\mathrm{Hiei}^{5)}$ report that on flash spectrograms taken at 1958 solar eclipse in South Pacific, one can notice the spicular structure even at levels as low as $1500 \mathrm{~km}$ above the top of the photosphere. Of course this is the lowest level ever reported, but still one can only say that the spicular structure starts from some heights below $1500 \mathrm{~km}$.

In this paper it is suggested that spicule structure starts from the zero $\mathrm{km}$ level, i.e., from the top of the photosphere. This rather unusual proposal is also based on the same eclipse observation and many possible consequences to be expected from this model will be discussed in some detail.

2. Eclipse Observation. As has already been reported elsewhere, in 1958 eclipse Suemoto and Hiei succeeded in photographing many flash spectra using a new type slitless spectrograph. This spectrograph using an oblique incidence technique ${ }^{6)}$ permits one to measure line profiles as well as total energies above the moon's limb on the same spectrum. On these flash spectra the present author measured the 
line profiles of suitable multiplets at many heights ranging from almost zero $\mathrm{km}$ up to $10,000 \mathrm{~km}^{7)}$ In particular above the height of $2000 \mathrm{~km}$ the measurement was made for a number of individual spicules or groups of spicules. Turbulent velocities were then derived by a method free from saturation effect, although precautions were paid not to use very saturated lines. The velocity thus found first increases from about $2 \mathrm{~km} / \mathrm{sec}$ to about $17 \mathrm{~km} / \mathrm{sec}$ as one ascends from the base of the chromosphere up to $2000 \mathrm{~km}$, and then the velocity tends to be constant up to the highest level of $10,000 \mathrm{~km}$. Velocities in the highest layers $(h>5000 \mathrm{~km})$ were determined by $\mathrm{H}$ and $\mathrm{K}$ lines of $\mathrm{Ca}$ II. Although each spicule looks to be transparent to these radiations at this level, it is very probable that at a little lower heights each spicule or each group of spicules would no more be transparent to these radiations. In other words, one would have to expect the same velocity of $17 \mathrm{~km} / \mathrm{sec}$ when one observes the sun in radial direction. Nevertheless, Goldberg, Mohler and Müller ${ }^{8)}$ obtained a velocity of about $6 \mathrm{~km} / \mathrm{sec}$ from the analysis of the $\mathrm{K}_{3}$ absorption profile appearing on the disk spectrum. If one wants to attribute this difference to an anisotropy, the anisotropy would have to be such that horizontal velocity is three times larger than the vertical. In the reversing layer this sort of anisotropy ${ }^{9)}$ is understandable, because the radiative layer acts as a barrier against the ascending motion from below, but in the chromosphere, where there is actually no barrier above it, this would be very unlikely.

The $\mathrm{K}_{3}$ line profile analysis cited above was made on photoelectric scanning of the solar spectrum and refers to an average spectrum within a slit area used. However, it is well known that there are many minute structures within this slit area. ${ }^{10)}$ In some parts of the disk $\mathrm{K}_{2}$ emissions are very intense and possibly $\mathrm{K}_{3}$ residual intensities are also much higher than elsewhere. Because of the insufficient resolution of the solar image, those two kinds of spectra, even if they had essentially different characters, might look alike on ordinary so called high resolution spectrograms. It is possible, therefore, that those parts with strong $\mathrm{K}_{2}$ emissions emit only broad emission lines without any central absorption, whereas the other parts do not emit any emission line at all and have only an absorption line profile with a sharp absorption core. As a reasonable assumption one could take the width of the sharp absorption core to be about three times the Doppler width at the top of the photosphere. If one takes this view the turbulence velocity of $6 \mathrm{~km} / \mathrm{sec}$ derived from the $K_{3}$ absorption would in fact be representative of a photospheric turbulence velocity of approximately $2 \mathrm{~km} / \mathrm{sec}$.

The width of the emission line should this time be close to the 
velocities used by Wilson and Bappu. ${ }^{11}$ From the measurement of Mohler the velocity was about $17 \mathrm{~km} / \mathrm{sec}$, in strikingly good agreement with the eclipse observation. This circumstance, however, would mean that the chromosphere is more or less transparent in radial observation of the sun. However, as the velocity decreases rapidly downwards below $2000 \mathrm{~km}$, this circumstance would only mean that the chromosphere or more precisely spicules or groups of spicules are more or less transparent down to $2000 \mathrm{~km}$. And optically thicker parts of the chromosphere do not show up on the radial observation because of their smaller velocities.

3. Extreme Ultraviolet Solar Spectrum. If this new interpretation of $\mathrm{K}_{3}$ absorption line is admitted, it would follow that the depth of the $K_{3}$ absorption as measured from the $K_{2}$ maxima should not exceed the residual intensity at $K_{1}$. This is confirmed by solar $\mathrm{H}$ and $\mathrm{K}$ lines themselves ${ }^{8)}$ and solar $\mathrm{Mg}$ II doublet near $2900 \mathrm{~A}^{{ }^{22}}$ The latter doublet has much more intense emissions than the former. On the other hand, $\mathrm{Mg}$ II lines have only very weak absorptions at their line centres. This is just the relative behaviour of these two doublets we would expect on our new interpretation.

In the case of Ly $\alpha$ in which $\mathrm{K}_{1}$ is practically zero, there should not be any absorption like $\mathrm{K}_{3}$. However, it is generally believed that although the sharp central absorption is due to the neutral hydrogen around the earth, weak wider absorption is due to the solar atmosphere just like $K_{3}$. If this is the case, our new interpretation cannot be applied to Ly $\alpha$ line. Since the chromosphere is very opaque to Ly $\alpha$ radiation, it is possible that Jefferies and Thomas' mechanism $^{13)}$ is working in this particular case. ${ }^{14)}$ Very large central absorption as was reported at the fourth COSPAR meeting in Warsaw by Tousey et $a l^{15)}$ looks to support this. However, in view of the big change in the number of telluric hydrogen depending upon the solar activity, it is still to be investigated whether the wider reversal in Ly $\alpha$ is really of solar origin or not. In fact, the equivalent widths of the central absorption as a whole were approximately $0.05 \mathrm{~A}$ and $0.3 \mathrm{~A}$ at 1959 flight $^{16)}$ and 1961 flight, $^{15)}$ respectively, whereas the number of telluric hydrogen was estimated to have increased by a factor of 8 according to Kockarts and Nicolet's model of the exosphere. ${ }^{17)}$

4. Stellar Spectra. The same circumstance as was described at the beginning of the last section should also hold in the spectra of the late type Ca II emission line stars. Since we have not much photometric data on these emissions, we have to content ourselves with more qualitative arguments. If there were any single star, for instance in Wilson and Bappu's table, ${ }^{11)}$ which has very strong emis- 
sions with very strong absorption cores, our interpretation would be wrong. Since no intensity measure as to the strength of $K_{3}$ absorption is given in this table, let us assume that "double" means a strong absorption and "single" means no absorption or very weak absorption. In order to exclude the absorption due to the circumstellar atmosphere, which constitutes a separate problem from the chromospheric $\mathrm{K}_{3}$ absorption, we subdivided the double peaked emissions into two groups depending upon whether the radial velocity of $\mathrm{K}_{3}$ absorption $(\Delta \mathrm{A})$ is larger than $6 \mathrm{~km} / \mathrm{sec}$ or not. The result is given in Table I. In Table I, the only exception to our expectation is $\alpha$

Table I. Stars with very strong $\mathrm{K}$ emissions do not have strong $\mathrm{K}_{3}$ absorptions of chromospheric origin.

\begin{tabular}{|c|c|c|c|}
\hline \multirow{3}{*}{ Int.* } & \multicolumn{3}{|c|}{ Number of stars } \\
\hline & \multicolumn{2}{|c|}{ Double } & \multirow[t]{2}{*}{ Single } \\
\hline & $\mathrm{CS}$ & Chr. & \\
\hline 5 & 1 & 0 & 10 \\
\hline 4 & 12 & $1^{\dagger}$ & 4 \\
\hline \multicolumn{4}{|c|}{$\begin{array}{l}\text { * } 5 \text { means } \mathrm{K}_{2} \text { emissions equal to or stronger than } \\
\text { the local continuum. } 4 \text { means } \mathrm{K}_{2} \text { emissions about } \\
50 \% \text { the local continuum. } \\
\dagger \alpha \mathrm{Tau} \text {, K5III. }\end{array}$} \\
\hline
\end{tabular}

Tau. However, this star is classified as K5III, and, even with a small radial velocity of $+2 \mathrm{~km} / \mathrm{sec}$ for an absorption component, it may be of circumstellar origin. It would seem, therefore, that not a single star was found which contradicts the new interpretation.

However, it has to be remarked that most of the stars listed in the last column are dwarf stars with only very small turbulent velocities. And it is sometimes difficult to resolve the double peaked emissions with a dispersion of $10 \mathrm{~A} / \mathrm{mm}$ used by Wilson and Bappu. In fact we found a number of double peaked emissions on stars listed as "single" in Wilson and Bappu's table. Historically speaking, Wilson and Bappu in turn found many double lined stars which had formally been classified as singles by Adams and Joy. ${ }^{18)}$ Our new observation was made by the $\mathrm{F} / 10$ camera of the coudé spectrograph of the Okayama Observatory, which has a dispersion of $4 \mathrm{~A} / \mathrm{mm}$. Among a number of stars $\varepsilon$ Eri, and 70 Oph A had double emission characteristics.

In particular, $\varepsilon$ Eri has a very interesting feature. The residual intensities of $\mathrm{H}_{2}$ and $\mathrm{K}_{2}$ of this star is rather high and $26 \%$ and $52 \%$, respectively, in units of the local continuum, and the relative intensity $\mathrm{K}_{2} / \mathrm{H}_{2}$ is very close to the theoretical ratio. This would indicate that 
the chromosphere is transparent to those radiations. Nevertheless, these lines have conspicuous $\mathrm{H}_{3}$ and $\mathrm{K}_{3}$ absorptions of about $5 \%$ in the same unit. With a current theory of $K_{3}$ formation such as Jefferies and Thomas' this feature would be very difficult to understand, because any theory which attributes the origin of the $K_{3} a b-$ sorption to the scattering processes would certainly need very high optical thickness. On our new interpretation this feature can as easily be understood as in other cases. Quantitative tests of the new interpretation based on photometric studies of these and similar stars are in progress.

5. A New Model of the Chromosphere. Based on the large difference in turbulence velocity from tangential observation and from radial observation of the solar chromosphere, a new interpretation of $\mathrm{K}_{3}$ absorption was proposed. The new interpretation proposes to understand the $\mathrm{K}$ line profile as an algebraic sum of the two different kinds of spectrum, one an absorption line spectrum from the top of the photosphere and the other an emission line spectrum from the spicules. It has been shown that this new interpretation does not contradict the solar and stellar spectra now available. In particular $\varepsilon$ Eri offers an evidence positively in favour of this interpretation, because this star has $\mathrm{H}_{3}$ and $\mathrm{K}_{3}$ absorption in spite of the fact that the optical thicknesses of the chromosphere in these lines are small.

As was described in $\S 3$ we have to admit that the case of $\mathrm{Ly}$ $\alpha$ constitutes a difficult problem. There is no doubt that the new interpretation cannot explain the wider absorption in the profile of Ly $\alpha$ if it is really due to the sun. However, it is to be remarked that this does not necessarily interfere with the new chromospheric model proposed here, as the chromosphere is optically very thick in this particular line.

We now like to propose a model of the chromosphere in which spicule structure starts from the top of the photosphere and extends upwards. It appears that the kinetic energy flow from the convection layer flows out from the top of the photosphere only in patches, and only above those patches there form spiclues protruding into the corona. The corona is heated by the kinetic energy flow through spicules, and the resulting heat is conducted back to spicules as well as to bare top of the photosphere above which there is no spicules, forming extremely thin transition layers covering both of those cool atmospheres. However, the transition layer on the bare top of the photosphere has to be optically (including radio) much thinner than the transition layer surrounding spicules, as was discussed by Moriyama $^{3)}$ and by Suemoto and Moriyama.) The resulting model would, therefore, be such that although spicules have sheath like transition 
layers, on the bare top of the photosphere there is practically no observable transition layer and merges more abruptly into the corona. The so-called interspicular space is, presumably, filled by the corona at all heights in the chromosphere, if the word "corona" is understood as an atmosphere with an electron density less than $10^{9} / \mathrm{cm}^{3}$.

\section{References}

1) R. G. Athay and D. H. Menzel: Astrophys. Journ., 123, 285 (1956).

2) R. G. Athay: Paris Symposium on Radio Astronomy (1959).

3) F. Moriyama: Ann. Tokyo Astr. Obs., 2nd Ser., 7, 127 (1961).

4) Z. Suemoto and F. Moriyama: 3rd COSPAR Symp., edited by Priester, Amsterdam, p. 800 (1963).

5) Z. Suemoto and E. Hiei: Publ. Astr. Soc. Japan, 14, 33 (1962).

6) Z. Suemoto and E. Hiei: Publ. Astr. Soc. Japan, 11, 122 (1959).

7) Z. Suemoto: Publ. Astr. Soc. Japan, 15 (1963), in press.

8) L. Goldberg, O. C. Mohler, and E. A. Müller: Astrophys. Journ., 129, 119 (1959).

9) C. W. Allen: Monthly Notices, 109, 343 (1949).

Z. Suemoto: Monthly Notices, 117, 2 (1957).

10) R. R. McMath, O. C. Mohler, A. K. Pierce, and L. Goldberg: Astrophys. Journ., 124, 1 (1956).

11) O. C. Wilson and M. K. Vainu-Bappu: Astrophys. Journ., 125, 661 (1957).

12) J. D. Purcell, A. Boggess III, and R. Tousey: IGY Rocket Report Series, No. 1, 198 (1958).

13) J. T. Jefferies and R. N. Thomas: Astrophys. Journ., 129, 401 (1959); 131, 695 (1960).

14) D. C. Morton and K. G. Widing: Astrophys. Journ., 133, 596 (1961).

15) R. Tousey, J. D. Purcell, W. E. Austin, D. L. Garrett, and K. G. Widing: 4th COSPAR Symp. (1963).

16) J. D. Purcell and R. Tousey: Mém. de la Société Royale des Sciences de Liège, 4, 274 (1961).

17) G. Kockart and M. Nicolet: Ann. de geophys., 18, 269 (1962).

18) W. S. Adams and A. H. Joy: Publ. Astr. Soc. Pacific, 43, 407 (1931). 